Chirurg 2018 $89: 930$

https://doi.org/10.1007/s00104-018-0655-9

Online publiziert: 17. Mai 2018

(c) Springer Medizin Verlag $\mathrm{GmbH}$, ein Teil von Springer Nature 2018

CrossMark

K. Hekmat ${ }^{1}$ C. J. Bruns ${ }^{2}$

'Klinik und Poliklinik für Herz- und Thoraxchirurgie, Universität zu Köln, Köln, Deutschland

${ }^{2}$ Klinik und Poliklinik Allgemein-, Viszeral- und Tumorchirurgie, Universität zu Köln, Köln, Deutschland

\title{
Lungenkrebs bei operierten Nichtrauchern
}

cher waren eher weiblich $(76 \%$ vs. $53 \%$, $p<0,001)$, Asiaten $(26,6 \%$ vs. $5,4 \%$, $p<0,001)$ und jünger (medianes Alter 67 vs. $70, p<0,001)$. Weitere Unterschiede zeigten sich bei einem höheren Anteil von Tumoren der Unterlappen $(38,8 \%$ vs. $31,2 \%, p<0,001)$ sowie niedrigeren „standardized uptake value“(SUV)-Werten im PET-CT (3,0 vs. 5,0, $p<0,001$ ). Bei den 498 Propensity-gematchten Paaren zeigten sich keine Unterschiede bei dem rezidivfreien ( $66 \%$ vs. $67 \%, p=0,66)$ sowie lungenkrebsspezifischen ( $84 \%$ vs. $81 \%, p=0,35)$ Überleben.

Fazit des Reviews. Die Ergebnisse der vorliegenden Studie mit 3232 Patienten zeigen eindrücklich, dass Nieraucher nach einer Lungenresektion wegen Bronchialkarzinom keinen günstigeren Verlauf im Vergleich zu Rauchern aufweisen. Die Rate an Lymphknotenmetastasen zeigte mit $21,1 \%$ bei den Nierauchern keinen signifikanten Unterschied (Raucher: 22,9\%), was für ein vergleichbares biologisches Verhalten der Bronchialkarzinome spricht. Im Stadium IA zeigte sich eine statistisch bessere Gesamtüberlebensrate bei den Nierauchern. Das lungenkrebsspezifische Überleben zeigte jedoch keine signifikanten Unterschiede, sodass der Unterschied bei der Gesamtüberlebensrate auf zusätzliche Nebenerkrankungen bei den Rauchern zurückzuführen ist. Der Anteil der Nieraucher scheint im thoraxchirurgischen Patientengut $\mathrm{zu}$ steigen. Da sich diese Studie nur mit resezierten Bronchialkarzinomen befasst, kann hieraus jedoch keine Aussage über die Inzidenz von Lungenkrebs bei Nierauchern abgeleitet werden. Molekulargenetische Analysen der Bronchi- alkarzinome könnten in Zukunft die Prognose von Nierauchern verbessern, da bei diesem Patientengut eine zielgerichtete, personalisierte Krebstherapie häufiger möglich ist.

\section{Korrespondenzadresse}

\section{Prof. Dr. K. Hekmat}

Klinik und Poliklinik für Herz- und Thoraxchirurgie, Universität zu Köln Kerpener Straße 62, 50931 Köln, Deutschland khosro.hekmat@uk-koeln.de

Interessenkonflikt. K. Hekmat und C. J. Bruns geben an, dass kein Interessenkonflikt besteht. 